$\mathbb{T}$ periodica polytechnica

Electrical Engineering

53/1-2 (2009) 3744

doi: 10.3311/pp.ee.2009-1-2.05

web: http://www.pp.bme.hu/ee

(c) Periodica Polytechnica 2009

RESEARCH ARTICLE

\section{Model predictive control for the hybrid primary circuit dynamics of a pressurized water nuclear power plant ${ }^{1}$}

Tamás Péni / Gábor Szederkényi

Received 2010-05-22

\begin{abstract}
In this paper, a model predictive controller is developed for controlling the main primary circuit dynamics of pressurized water nuclear power plants during load-change transients. The hybrid model of the plant is successfully embedded into a nonhybrid discrete time LPV form. The designed controller is able to handle the hard constraints for the state and input variables while keeping the plant stable and producing satisfactory timedomain behavior.
\end{abstract}

\section{Keywords}

model predictive control $\cdot$ nuclear power plant $\cdot$ hybrid systems $\cdot$ linear matrix inequalities

\section{Acknowledgement}

This research was partially supported by the Hungarian grants No. T042710, F046223, which are gratefully acknowledged.

Tamás Péni

Gábor Szederkényi

Systems and Control Laboratory of the Computer and Automation Research Institute, MTA, P.O. Box 63, H-1518 Budapes, Hungary

e-mail: pt@scl.sztaki.hu

\section{Introduction}

The paper describes a model predictive control scheme for the primary circuit system of the Paks Nuclear Power Plant (Paks NPP) located in Hungary. The Paks NPP was founded in 1976 and started its operation in 1981. The plant operates four VVER-440/213 type reactor units with a total nominal (electrical) power of $1860 \mathrm{MWs}$. About 40 percent of the electrical energy generated in Hungary is produced here. Considering the load factors, the Paks units belong to the leading ones in the world and have been among the top twenty-five units for years.

The main motivations behind the present work are the following. Firstly, due to the continuous reconstruction of the measurement equipment and the information infrastructure, more and more measurement data are available in good quality. This fact allowed us the control-oriented modeling and parameter identification of the primary circuit dynamics [11]. Secondly, the present control configuration of the plant is a distributed scheme, where the controllers are tuned individually. The current operation of the system in the neighborhood of the prescibed operating points is satisfactory, but studies and simulations show that the dynamic behavior during bigger transients mainly caused by load changes can be improved by applying a multivariable controller. Thirdly, another motivating fact is a previous work: the successful modeling, identification [11], controller design [10] and implementation of the pressure control loop in the primary circuits of units 1, 3 and 4 of the plant. Using this model-based design, the precise stabilization of the primary loop pressure was a key factor in the safe increase of the average thermal power of the units by approximately $1-2 \%$ in 2005 .

The main aim of this paper is to propose an integrated controller, which eliminates some imperfections of the present control architecture. This new controller belongs to the model predictive control (MPC) scheme. The MPC is an optimization based control method, where, assuming discrete time case, an open loop optimal control problem is solved in each sampling

\footnotetext{
${ }^{1}$ This paper revises and extends the results of the paper 'LMI-based model predictive control for the hybrid primary circuit dynamics of a pressurized water nuclear power plant' presented in the European Control Conference, 2007, Kos, Greece.
} 
instant, using the actual state as initial state, and the first element of the obtained control sequence is applied to the plant. For the theoretical background of MPC see e.g. [7], [6]. The model predictive approach has several advantages: it is able to handle complex systems where off-line computation of the control law is difficult, and in contrast to other techniques it is able to handle hard constraints prescribed for the states and control inputs.

The paper is organized as follows. After the introduction in section 1, a nonlinear hybrid model of the plant is constructed. Section 2 contains the main control objectives. In section 2.A, the LMI-based MPC method proposed by Kothare et.al [5] is introduced, which is slightly modified in section 2.B. The original and the modified methods are applied and tested on the nonlinear system model by numerical simulations. The simulation results are presented and analyzed in section 4 . The most important conclusions are summarized in section 5.

\section{System model}

\subsection{Overall system description}

The liquid in the primary circuit is circulated at a high speed by powerful circulation pumps, and it is under high pressure in order to avoid boiling. The energy generated in the reactor is transferred by the primary circuit to the liquid in the steam generator making it boiling. The generated secondary circuit vapor is then transferred to the turbines.

Fig. 1 shows the flowsheet of the primary circuit in Paks NPP, where the main equipments are the reactor, the steam generator(s), the main circulating pump(s), the pressurizer and their connections are depicted. The sensors that provide on-line measurements are also indicated in the figure by small full rectangles. The controllers are denoted by double rectangles, their input and output signals are shown by dashed lines.

\subsection{Continuous time state-space model}

The dynamic model of the process has been constructed using a systematic modeling approach proposed in [4]. The detailed modeling and model identification procedure has been described in [3]. The continous time state-space model of the system is the following

$$
\begin{aligned}
\frac{d N}{d t} & =\frac{\beta}{\Lambda}\left(\rho_{\max }-\left(p_{1} v^{2}+p_{2} v+p_{3}\right)\right) N+S \\
\frac{d T_{P C}}{d t} & =\frac{1}{c_{p, P C} M_{P C}}\left[c_{p, P C} m_{\text {in }}\left(T_{P C, I}-T_{P C, C L}\right)+\right. \\
& W_{R}-6 \cdot K_{T, S G_{1}}\left(T_{P C}-T_{S G}\right)- \\
& \left.-W_{\text {loss }, P C}\right] \\
\frac{d T_{S G}}{d t} & =\frac{1}{c_{p, S G}^{L} M_{S G}}\left[c_{p, S G}^{L} m_{S G} T_{S G, S W}-\right. \\
& c_{p, S G}^{V} m_{S G} T_{S G}-m_{S G} E_{\text {evap }, S G}+ \\
& \left.+K_{T, S G_{2}}\left(T_{P C}-T_{S G}\right)-W_{\text {loss }, S G}\right]
\end{aligned}
$$

$$
\begin{aligned}
& \left.\frac{d T_{P R}}{d t}\right|_{m_{P R}>0}= \\
& \frac{1}{c_{p, P R} M_{P R}}\left[c_{p, P C} m_{P R} T_{P C, H L}-c_{p, P R} m_{P R} T_{P R}-\right. \\
& \left.-W_{\text {loss }, P R}+W_{\text {heat }, P R}\right] \\
& \left.\frac{d T_{P R}}{d t}\right|_{m_{P R} \leq 0}=\frac{1}{c_{p, P R} M_{P R}}\left[-W_{\text {loss }, P R}+W_{\text {heat }, P R}\right]
\end{aligned}
$$

where $W_{R}=c_{\psi} N$. The measurable variables and constant parameters of the model are summarized in Table 1 The abbreviations $R, P C, S G, P R$ refer to the reactor, primary circuit, steam generator, and pressurizer, respectively.

The mass flow $m_{P R}$ (which makes the system dynamics hybrid) from the primary circuit to the pressurizer (or backward) can be written as

$$
m_{P R}=-V_{P C}^{0} c_{\phi, 1} \frac{d T_{P C}}{d t}
$$

We assume that the variables $m_{i n}, T_{P C, I}, m_{S G}$ and $T_{S G, S W}$ are known and constant which is an acceptable approximation of reality from a control point of view. The control inputs are the rod position $(v)$ and the heating power of the pressurizer $\left(W_{\text {heat }, P R}\right)$. Instead of $v$ we introduce $v=\left(p_{1} v^{2}+p_{2} v+p_{3}\right) N$ as a new control input, since Eq. (1) depends linearly on $v$. This can be done, since the polynomial $p(v)=p_{1} v^{2}+p_{2} v+p_{3}$ is monotonously increasing, thus invertible: $v=p^{-1}(v / N)$. The constraints prescribed for $v$ can be transformed into equivalent constraints prescribed for $v$ as follows: $v_{\min } \leq v \leq v_{\max }$ $\Leftrightarrow N_{\min } p\left(v_{\min }\right) \leq v \leq N_{\min } p\left(v_{\max }\right)$, where $p\left(v_{\min }\right)<0<$ $p\left(v_{\max }\right)$ and $N_{\min }$ is a physical limit for which $0<N_{\min } \leq N$ always holds.

Since $\frac{d T_{P C}}{d t}$ does not depend on $m_{P R}$, Eq. (6) can be substituted into Eqs. (1)-(5) without producing algebraic loop. Carrying out this simple manipulation and centering the model around a predefined operating point $\left(\bar{N}, \bar{T}_{P C}, \bar{T}_{S G}, \bar{T}_{P R}, \bar{v}, \bar{W}_{\text {heat }, P R}\right)$ the dynamic model can be rewritten in the following more compact form:

$$
\begin{aligned}
\dot{s} & =A s+B u_{1} \\
\left.\dot{z}\right|_{m_{P R}>0} & =\left(a_{s}^{T}+z p^{T}+s_{2} q^{T}\right) s+a_{z} z+b u_{2} \\
\left.\dot{z}\right|_{m_{P R} \leq 0} & =b u_{2} \\
m_{P R} & =m_{s}^{T} s
\end{aligned}
$$

where $s=\left[N-\bar{N}, T_{P C}-\bar{T}_{P C}, T_{S G}-\bar{T}_{S G}\right], z=T_{P R}-\bar{T}_{P R}$, $u_{1}=v-\bar{v}, u_{2}=W_{\text {heat }, P R}-\bar{W}_{\text {heat }, P R}$ and $A, B, a_{x}, a_{z}, p, q, b$, $m_{s}$ are constant matrices, vectors of appropriate dimensions. If the state variables $s_{2}$ and $z$ in the nonlinear terms are considered as time-varying parameters $\rho_{1}=s_{2}, \rho_{2}=z$ the equations above 


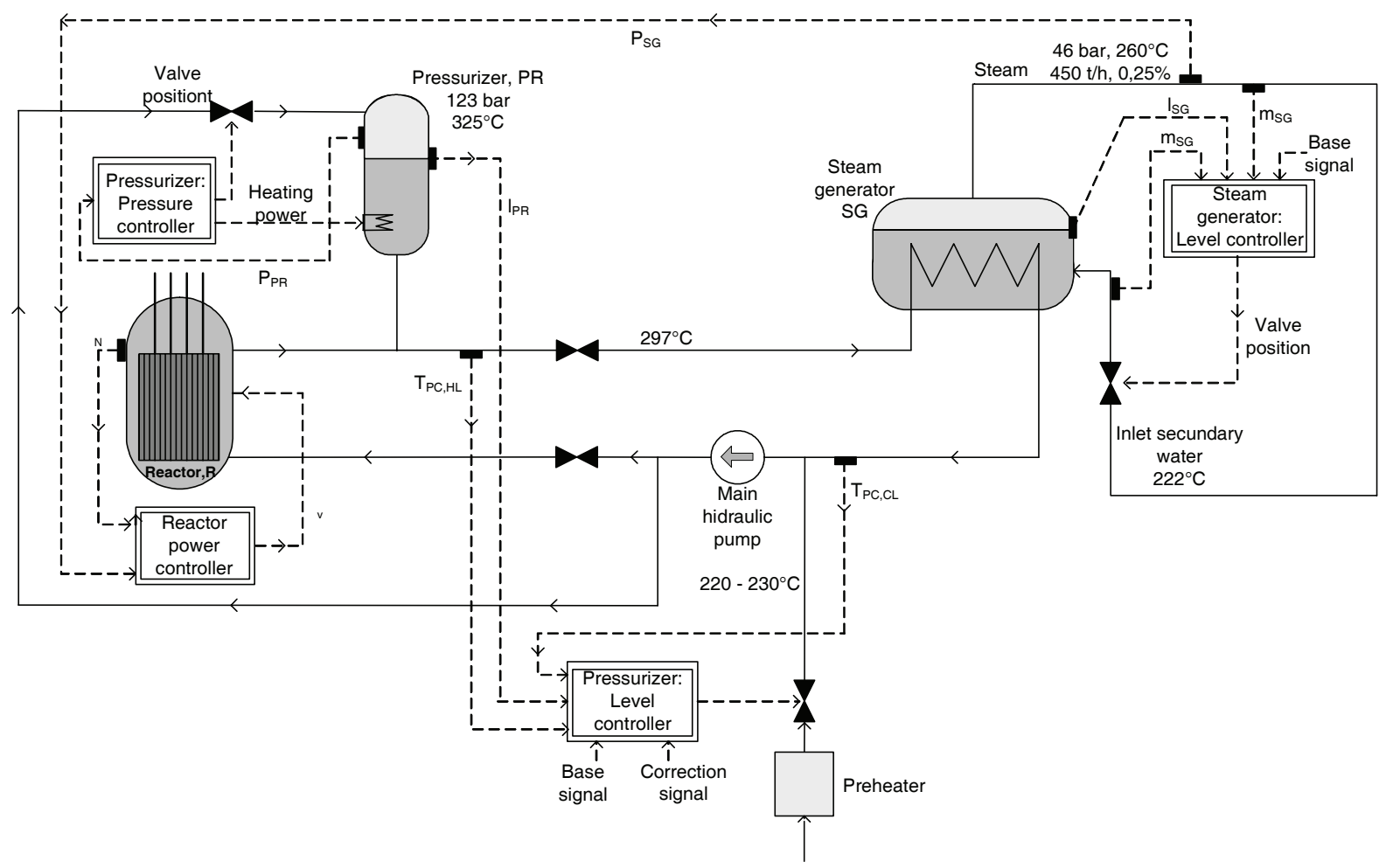

Fig. 1. The flowsheet of the primary circuit

take the following hybrid-LPV form:

$$
\begin{aligned}
\left.\dot{x}\right|_{m_{P R}>0} & =\left(A_{c, 0}+\rho_{1} A_{c, 1}+\rho_{2} A_{c, 2}\right) x+B_{c} u \\
& =A_{c}(\rho) x+B_{c} u \\
\left.\dot{x}\right|_{m_{P R} \leq 0} & =A_{c, 3} x+B_{c} u \\
m_{P R} & =m^{T} x
\end{aligned}
$$

where $A_{c, 0}, A_{c, 1}, A_{c, 2}, A_{c, 3}$ and $B_{c}$ are constant matrices, and $x=\left[\begin{array}{ll}s & z\end{array}\right]^{T}, m=\left[\begin{array}{ll}m_{s}^{T} & 0\end{array}\right]^{T}$.

\subsection{Discrete time model}

To apply model predictive control, the continuous model of the system has to be discretized. If $m_{P R} \leq 0$ the dynamics is linear, so it can be easily transformed into discrete-time. We have to concentrate only on the first, parameter varying subsystem. Since the parameters $\rho_{1}$ and $\rho_{2}$ vary relatively slowly in time, the discretization can be performed in the following way: at a time instant $t_{k}$ the parameters are fixed and the linear system obtained is discretized by computing its solution under constant input $u_{k}$ :

$$
\begin{aligned}
x\left(t_{k}+T_{s}\right) & \approx \\
x_{k+1} & =A_{d}(k) x_{k}+B_{d}(k) u_{k} \\
A_{d}(k) & =e^{A_{c}\left(\rho\left(t_{k}\right)\right) T_{s}} \\
B_{d}(k) & =\int_{0}^{T_{s}} e^{A_{c}\left(\rho\left(t_{k}\right)\right)\left(T_{s}-\tau\right)} B_{c} d \tau
\end{aligned}
$$

Fortunately, the computation of $A_{d}(k), B_{d}(k)$ can be simplified if the special structure of the matrices $A_{c, i}$ is exploited. By calculating the spectral decomposition of $A_{c}(\rho)$ symbolically (with parameters $\left.\rho_{1}, \rho_{2}\right)$ it can be seen that its eigenvalues are all distinct and do not depend on the parameters. Thus

$$
e^{A_{c}\left(\rho\left(t_{k}\right)\right) T_{s}}=V\left(\rho\left(t_{k}\right)\right) \operatorname{diag}\left(e^{\lambda_{i}}\right) V\left(\rho\left(t_{k}\right)\right)^{-1}
$$

where $V(\rho)=V_{0}+\rho_{1} V_{1}+\rho_{2} V_{2}$. Continuing the analysis, we can see that the eigenvectors $V(\rho)$ are also of special form, which enables us to express the matrices $A_{d}(k), B_{d}(k)$ as follows:

$$
\begin{aligned}
A_{d}(k) & =A_{d, 0}+\rho_{1} A_{d, 1}+\rho_{2} A_{d, 2} \\
A_{d, 0} & =e^{A_{c, 0} T_{s}} \\
A_{d, i} & =e^{\left(A_{c, 0}+A_{c, i}\right) T_{s}}-A_{d, 0}, i=1,2 \\
B_{d}(k) & =B_{d, 0}+\rho_{1} B_{d, 1}+\rho_{2} B_{d, 2} \\
B_{d, 0} & =\int_{0}^{T_{s}} e^{A_{c, 0}\left(T_{s}-\tau\right)} B_{c} d \tau \\
B_{d, i} & =\int_{0}^{T_{s}} e^{\left(A_{c, 0}+A_{c, i}\right)\left(T_{s}-\tau\right)} B_{c} d \tau-B_{d, 0}
\end{aligned}
$$

Thus, the hybrid LPV form 8 is preserved after the discretization:

$$
\begin{aligned}
&\left.x_{k+1}\right|_{m_{P R}>0}=\left(A_{d, 0}+\rho_{1} A_{d, 1}+\rho_{2} A_{d, 2}\right) x_{k}+ \\
&(\left(B_{d, 0}+\rho_{1} B_{d, 1}+\rho_{2} B_{d, 2}\right) u_{k} \\
&\left.x_{k+1}\right|_{m_{P R} \leq 0}=A_{d, 3} x_{k}+B_{d, 3} u_{k} \\
& m_{P R}=m^{T} x_{k}
\end{aligned}
$$

The MPC framework applied later requires the system to be in polytopic form [5]. For this, we have to introduce upper and lower bounds for the parameters $\underline{\rho}_{1} \leq \rho_{1} \leq \bar{\rho}_{1}, \underline{\rho}_{2} \leq \rho_{2} \leq \bar{\rho}_{2}$, 
Tab. 1. Measured variables and constant parameters of the model (Notations: state, input, otput, disturbance)

\begin{tabular}{|c|c|c|c|c|c|}
\hline Identifier & Variable & Type & Identifier & Parameter & Unit \\
\hline$N$ & R neutron flux & $\mathrm{s}$ & $\left(p_{1}, p_{2}, p_{2}\right)$ & control rod parameters & $\mathrm{R}$ \\
\hline$v$ & $\mathrm{R}$ control rod position & $\mathrm{i}$ & $\rho_{\max }$ & maximum reactivity & $\mathrm{R}$ \\
\hline$W_{R}$ & $\mathrm{R}$ reactor power & 0 & $S$ & zero neutron flux & $\mathrm{R}$ \\
\hline$m_{i n}$ & PC inlet mass flow rate & $\mathrm{i}$ & $c_{p, P C}$ & specific heat & $\mathrm{PC}$ \\
\hline$T_{P C, I}$ & PC inlet temperature & $d$ & $M_{P C}$ & water mass & PC \\
\hline$T_{P C, C L}$ & PC cold leg temperature & (s) & $K_{T, S G_{1,2}}$ & heat transfer coefficients & $\mathrm{PC}, \mathrm{SG}$ \\
\hline$T_{P C, H L}$ & PC hot leg temperature & $(\mathrm{s})$ & $T_{\text {out }}$ & containment temperature & $\mathrm{PC}$ \\
\hline$p_{P R}$ & PR pressure & $0,(\mathrm{~s})$ & $M_{S G}$ & water mass & SG \\
\hline$T_{P R}$ & PR temperature & $\mathrm{s}$ & $W_{\text {loss }, P C}$ & heat loss & $\mathrm{PC}$ \\
\hline$\ell_{P R}$ & PR water level & $0,(s)$ & $W_{l o s s, S G}$ & heat loss & SG \\
\hline$W_{\text {heat }, P R}$ & PR heating power & i & $c_{p, S G}^{L}$ & liquid specific heat & SG \\
\hline$m_{S G}$ & SG mass flow rate & $d$ & $c_{p, S G}^{V}$ & vapor specific heat & SG \\
\hline$T_{S G, S W}$ & SG inlet water temperature & $d$ & $c_{p, P R}$ & liquid specific heat & PR \\
\hline$p_{S G}$ & SG steam pressure & 0 & $W_{\text {loss }, P R}$ & heat loss & PR \\
\hline
\end{tabular}

to be able to express the dynamics in the required form.

$$
\begin{aligned}
& x_{k+1}^{m_{P R}>0}=A(k) x_{k}+B(k) u_{k} \quad[A(k), B(k)] \in \Omega \\
& \Omega=\operatorname{Co}\left\{\left[A_{1}, B_{1}\right], \ldots,\left[A_{4}, B_{4}\right]\right\} \\
& A_{i}=A_{d, 0}+\delta_{i, 1} A_{d, 1}+\delta_{i, 2} A_{d, 2} \\
& B_{i}=B_{d, 0}+\delta_{i, 1} B_{d, 1}+\delta_{i, 2} B_{d, 2} \\
& \delta_{i, 1} \in\left\{\underline{\rho}_{1}, \bar{\rho}_{1}\right\} \delta_{i, 2} \in\left\{\underline{\rho}_{2}, \bar{\rho}_{2}\right\} \\
& \left.x_{k+1}\right|_{m_{P R} \leq 0}=A_{d, 3} x_{k}+B_{d, 3} u_{k} \\
& m_{P R}=m^{T} x_{k}
\end{aligned}
$$

Notice that, if we complete the set of corner points of $\Omega$ with the system $\left[A_{5}, B_{5}\right]=\left[A_{d, 3}, B_{d, 3}\right]$ the hybrid dynamics above can be embedded into the following non-hybrid LPV system:

$$
\begin{aligned}
& x_{k+1}=A(k) x_{k}+B(k) u_{k} \quad[A(k), B(k)] \in \Omega \\
& \Omega=\operatorname{Co}\left\{\left[A_{1}, B_{1}\right], \ldots,\left[A_{4}, B_{4}\right],\left[A_{5}, B_{5}\right]\right\}
\end{aligned}
$$

where $\mathrm{Co}(\cdot)$ denotes the convex hull of its arguments. This can be easily checked by considering the following convex combinations

$$
A(k)=\sum_{i=1}^{5} \gamma_{1} A_{i}, \quad B(k)=\sum_{i=1}^{5} \gamma_{i} B_{i}, \quad \sum_{i=1}^{5} \gamma_{i}=1
$$

with $\sum_{i=1}^{4} \gamma_{i}=1, \gamma_{5}=0$ if $m_{P R}>0$ and $\gamma_{1}=\gamma_{2}=\gamma_{3}=$ $\gamma_{4}=0, \gamma_{5}=1$ if $m_{P R} \leq 0$.

\section{Controller design}

\subsection{Control goals, assumptions and constraints}

In the present control configuration, the neutronflux and the heating in the pressurizer are controlled separately. This performs quite well in the neighborhood of the prescribed steady states, but during large load changes, the temperature in the pressurizer usually slightly goes out of the required optimal operating interval. Therefore, the goal of the controller design is to obtain such a controller that - first of all - keeps all the predefined hard constraints for the state and input variables and secondly, it produces a satisfactorily quick load change transient. More precisely, the aim is to design an integrated controller, which steers the system from one operating point to another, so that

- the settling time of the neutron flux $N$ be as small as possible

- the temperature change in the pressurizer be at most $1 \mathrm{~K}$ during the transient

- the control inputs $v, W_{\text {heat }, P R}$ satisfy the given hard, physical constraints, coming from the limited heating energy at the pressurizer.

\subsection{Model predictive control using linear matrix inequalities}

The control method we intend to apply is based on the MPC procedure proposed by Morari et.al in [5] and [2]. First, this procedure will be introduced briefly.

Suppose the system to be controlled is given in the form of (14) with an output equation $y(k)=C x(k), y(k) \in \mathbb{R}^{n_{y}}$. Concentrating on the robust regulation problem, i.e. steering the state from an arbitrary initial value $x_{0}$ to the origin the MPC solution proposed by [5] involves the following min-max optimization problem:

$$
\begin{array}{r}
\min _{u_{k+i \mid k}, i=0,1, \ldots, m[A(k+i), B(k+i)] \in \Omega} J_{k}^{\infty}, \\
J_{k}^{\infty}=\sum_{i=0}^{\infty}\left(x_{k+i \mid k}^{T} Q_{1} x_{k+i \mid k}+u_{k+i \mid k}^{T} R u_{k+i \mid k}\right)
\end{array}
$$

where $J_{k}^{\infty}$ is a prescribed infinite horizon cost function and $x_{k+i \mid k}, u_{k+i \mid k}$ denote the predicted state and control action at time instant $k+i$, both based on the state measurement $x_{k}=$ $x_{k \mid k}$. This optimization has to be performed at each sampling instant with the actual state measurements to obtain the next control input. Since this problem is computationally demanding, the following idea has been applied: if it is possible to find a quadratic function $V\left(x_{k}\right)=x_{k}^{T} P_{k} x_{k}$, which gives an upper bound on the robust performance objective $J_{k}^{\infty}$, the min-max problem can be replaced by a minimization of this quadratic 
function over the sequences of possible control moves. This can be easily solved by using linear matrix inequalities [9]. For $V\left(x_{k}\right)$ to be an upper bound it has to satisfy the following inequality [5]:

$$
\begin{array}{r}
V\left(x_{k+i+1 \mid k}\right)-V\left(x_{k+i \mid k}\right) \leq \\
-\left(x_{k+i \mid k}^{T} Q_{1} x_{k+i \mid k}+u_{k+i \mid k}^{T} R u_{k+i \mid k}\right)
\end{array}
$$

Since in this case

$$
-V\left(x_{k}\right) \leq-J_{k}^{\infty} \Rightarrow \max _{[A(k+i), B(k+i)] \in \Omega} J_{k}^{\infty} \leq V\left(x_{k}\right)
$$

holds. Using this upper bound, (16) can be replaced by the following simpler problem:

$$
\begin{array}{r}
\min _{u_{k+i \mid k}, i=0,1, \ldots, m[A(k+i), B(k+i)] \in \Omega} J_{k}^{\infty} \leq \\
\min _{u_{k+i \mid k}, i=0,1, \ldots} V\left(x_{k}\right)=\min _{u_{k+i \mid k}, i=0,1, \ldots} x_{k}^{T} P_{k} x_{k}
\end{array}
$$

If $u_{k+i \mid k}$ is chosen to be $u_{k+i \mid k}=F_{k} x_{k+i \mid k}$ it can be shown (see [5]) that the solution $\left(F_{k}, P_{k}\right)$ of (19) can be obtained in the following form:

$$
F_{k}=F=Y Q^{-1}, \quad P_{k}=P=\gamma Q^{-1}
$$

where $Q>0, \gamma>0, Y$ are the solutions of the following linear objective minimization problem:

$$
\min _{\gamma, Q, Y} \gamma
$$

subject to

$$
\left[\begin{array}{ll}
1 & x^{T} \\
x & Q
\end{array}\right] \geq 0
$$

and

$$
\left[\begin{array}{cccc}
Q & Q A_{j}^{T}+Y^{T} B_{j}^{T} & Q Q_{1}^{\frac{1}{2}} & Y^{T} R^{\frac{1}{2}} \\
A_{j} Q+B_{j} Y & Q & 0 & 0 \\
Q_{1}^{\frac{1}{2}} Q & 0 & \gamma I & 0 \\
R^{\frac{1}{2}} Y & 0 & 0 & \gamma I
\end{array}\right] \geq 0
$$

where $x=x_{k}$ and (23) is equivalent to the condition (17). If there are constraints prescribed for the input and/or output, they can be easily taken into consideration by expressing them as LMIs and attaching them to the constraints 22, 23) in the optimization problem above. For example, consider the peak bounds prescribed on each component of the input $u_{k}$, i.e.

$$
\left|u_{j, k+i \mid k}\right| \leq u_{j, \max }, \quad j=1 . . n_{u}
$$

where $n_{u}$ is the number of inputs. This holds if

$$
\left[\begin{array}{cc}
X & Y \\
Y^{T} & Q
\end{array}\right] \geq 0, \quad X_{j j} \leq u_{j, \max }^{2}
$$

The component-wise peak bounds on the outputs involve further LMI constraints, that can be given as:

$$
\begin{gathered}
{\left[\begin{array}{cc}
Q & \left(A_{j} Q+B_{j} Y\right)^{T} C_{l}^{T} \\
C_{l}\left(A_{j} Q+B_{j} Y\right) & y_{l, \text { max }}^{2} I
\end{array}\right] \geq 0} \\
j=1, \ldots, L, \quad l=1 \ldots n_{y}
\end{gathered}
$$

where $C_{l}$ is the $l$-th row of $C$. For further details see [5] and [1]. The following Theorem summarizes the main result of [5]:

Theorem 1 If at any time $k$ there exists $Q, Y, \gamma$ and $F_{k}=$ $Y Q^{-1}$ solving the problem (21) then

- the control input $F_{k} x_{k+i \mid k}$ will be feasible for all times $i>0$

- the control policy given by the MPC procedure stabilizes the polytopic system and satisfies the prescribed input/output constraints.

\subsection{Implementation issues}

The MPC procedure above assumes that the control gain $F_{k}$ is available at the same time instant $k$, when the measurement $x_{k}$ is taken. This means that the computation time of $F_{k}$ (the time needed to solve the optimization problem above) is neglected, or assumed to be negligible compared to the sampling time $T_{S}$. Unfortunately, in our case this assumption does not hold. The reactor dynamics is sampled with $T_{s}=1 \mathrm{sec}$, while the solution of the LMIs 23, 25] and 26] takes minimum $0.7 \mathrm{sec}$ maximum $1.02 \mathrm{sec}$ (depending on the actual state measurement $x_{k}$ ). Decreasing the frequency of the controller update is not enough to solve this problem, since in itself it does not provide more time for computation. The following procedure is proposed instead: new controller is designed only at each $M$-th time instant; between two controller design steps the feedback gain is calculated as follows:

$$
\begin{aligned}
\tilde{F}_{i} & =F_{k-M} \text { if } i-k \leq l \\
\tilde{F}_{i} & =F_{k-M}+\frac{i-k-l}{M-l}\left(F_{k}-F_{k-M}\right) \text { if } i-k>l \\
& i=k, \ldots, k+M-1, k=n \cdot M, \forall n
\end{aligned}
$$

After measuring $x_{k}$ at the step $k$ there are $l$ time steps $\left(l \cdot T_{s} s e c\right)$ to determine the new control input $F_{k}$. During this time the system is controlled by the previous controller $F_{k-M}$. After having determined $F_{k}$ we give it to the plant step by step, according to the interpolating rule above. The cause why $F_{k}$ is not applied immediately is the observation that our system is sensitive to the change of the control gain. This means that small changes in $F$ cause undesired oscillations in the system trajectories, especially in $m_{P R}$. The linear interpolation attenuates this effect.

The modified control strategy does not necessarily inherit the advantageous properties (constraint satisfaction, feasibility, stb.) of the original control policy. To ensure the feasibility of $\tilde{F}_{i}$ the 
following slightly conservative method has been chosen: at time $k$ the gain $F_{k}$ is designed so that the controlled system is stable and satisfies the constraints for all convex combinations of the new and the previous control gains, i.e.:

$$
\tilde{F}=\alpha F_{k-M}+(1-\alpha) F_{k}
$$

Replacing the control input $u_{k}=F_{k} x_{k+i \mid k}$ with $u_{k}=\tilde{F} x_{k+i \mid k}$ and following the same argument as [5] the new LMI conditions can be easily recalculated. The LMIs 23, 25 , and (26) have to be replaced by the LMIs (30, 31) and (32), respectively, which are defined as follows:

LMIs 23) and

$$
\left[\begin{array}{cccc}
Q & Q A_{j}^{T}+Q \bar{F}^{T} B_{j}^{T} & Q Q_{1}^{\frac{1}{2}} & Q \bar{F}^{T} R^{\frac{1}{2}} \\
A_{j} Q+B_{j} \bar{F}^{T} Q & Q & 0 & 0 \\
Q_{1}^{\frac{1}{2}} Q & 0 & \gamma I & 0 \\
R^{\frac{1}{2}} \bar{F} Q & 0 & 0 & \gamma I
\end{array}\right] \geq 0,
$$$$
j=1 . . L
$$

LMIs (25) and

$$
\left[\begin{array}{cc}
X & \bar{F} Q \\
Q \bar{F}^{T} & Q
\end{array}\right] \geq 0, \quad X_{j j} \leq u_{j, \text { max }}^{2}
$$

LMIs 26) and

$$
\left[\begin{array}{cc}
Q & \left(A_{j} Q+B_{j} \bar{F} Q\right)^{T} C_{l}^{T} \\
C\left(A_{j} Q+B_{j} \bar{F} Q\right) & y_{l, \text { max }}^{2} I
\end{array}\right] \geq 0
$$

where $F=F_{k}, P=P_{k}, \bar{F}=F_{k-M}$. It can be seen that the new sets of LMIs, beside the original $F$-dependent inequalities, contain further LMIs, which depend on the previous control gain $\bar{F}$. For further details see [8]. The modified control problem, therefore, can be handled in the same way as the original one, except that it involves more LMI constraints.

The properties of the modified control policy can be summarized in the following theorem.

Theorem 2 a The control gain $F_{k}$ defines a feasible control policy for all time $t>k$.

$b$ The control law obtained by using the modified MPC algorithm stabilizes the closed loop system, and satisfies the input and state constraints.

Proof 1 The proof is based on showing that the control policy

$$
\begin{aligned}
\tilde{F}_{i} & =F_{k-M}+\frac{i-k-l}{M-l}\left(F_{k}-F_{k-M}\right) \\
i & =k \ldots k+M-1 \\
\tilde{F}_{i} & =F_{k} \quad i \geq k+M
\end{aligned}
$$

(which is the control policy 28] extended to infinite horizon) is feasible at all time. The details of the proof can be found in [8].
Remark 1 Although 30, 31 and 32 contain more LMIs than 23, 25] and 26), the number of decision variables is the same in the two optimization problems. Therefore the modified control policy does not require significantly more computation time than the original one.

Remark 2 Our algorithm requires high computational power only at the M-th time steps, when the LMIs (30), (31) and 32) are solved. In other sampling instants a much simpler (lowlevel) computing hardware is enough to realize the interpolation (28). Therefore the modified algorithm can be implemented on a computer architecture depicted in Fig. 4. When there is no need for the high capacity computer, it can be used to solve other tasks related to the power plant.

\section{Simulation results}

The control method was tested on an identified model of the pressurized water nuclear power plant. The dynamics was sampled with $T_{S}=1 \mathrm{~s}$ and it was centered around the operating point belonging to $\bar{N}=100 \%$ and $\bar{T}_{P R}=599 \mathrm{~K}$. The remaining two state variables were determined by substituting $\bar{N}, \bar{T}_{P R}$ into (1)-(5) and solving the equations for 0 . The steady-state values obtained for $T_{P C}, T_{S G}$ and the control inputs were as follows:

$$
\begin{aligned}
& \bar{T}_{P C}=553.7398 \quad \bar{T}_{S G} \quad=530.3435 \\
& \bar{u}_{1}=4.5312 \quad \bar{u}_{2} \quad=1.6823
\end{aligned}
$$

In the simulation we examined the behavior of the plant under load increase, i.e. when the states are steered to the origin (to the steady state (34) ) from a workpoint belonging to a lower neutron flux. In our case $\bar{N}(0)=85 \%$ and

$$
\bar{T}_{P C}(0)=548.9279 \quad \bar{T}_{S G}(0)=529.4117
$$

In the centered model these values are equivalent to the following initial state:

$$
x(0)=-\left[\begin{array}{llll}
15 & 4.8119 & 0.9318 & 0
\end{array}\right]^{T}
$$

Since we had constraints prescribed for $N$ and $T_{P R}$, these two state variables were chosen as outputs, i.e.:

$$
C=\left[\begin{array}{llll}
1 & 0 & 0 & 0 \\
0 & 0 & 0 & 1
\end{array}\right]
$$

The constraints were as follows:

$$
\begin{array}{rr}
-20 \leq x_{1} \leq 20 & (\bar{N}-10 \leq N \leq \bar{N}+10) \\
-1 \leq x_{4} \leq 1 & \left(\bar{T}_{P R}-1 \leq T_{P R} \leq \bar{T}_{P R}+1\right)
\end{array}
$$

The control inputs always have to be in a physically realizable range. This means for $W_{\text {heat }} P R$ to be between 0 and 3.6 and for $v$ to be between -15 and 15 . Since in the MPC framework the limits have to be symmetric to 0 we used the following constraints:

$$
\begin{aligned}
& -10 \leq u_{1} \leq 10 \\
& -\bar{u}_{2} \leq u_{2} \leq \bar{u}_{2} \quad\left(0 \leq W_{\text {heat }, P R} \leq 3.3646\right)
\end{aligned}
$$


N

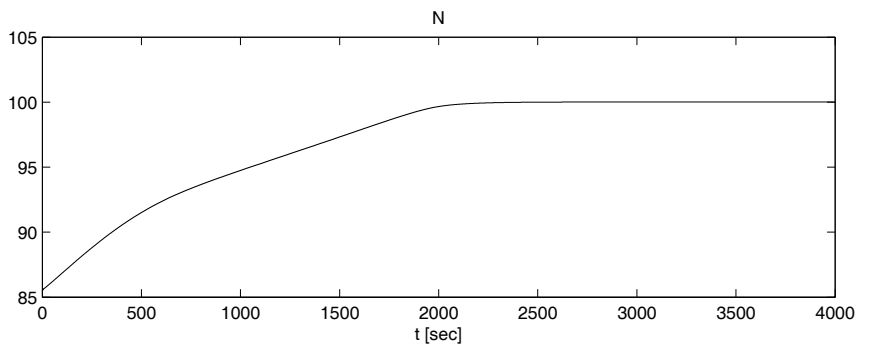

$\mathrm{T}_{\mathrm{PC}}$

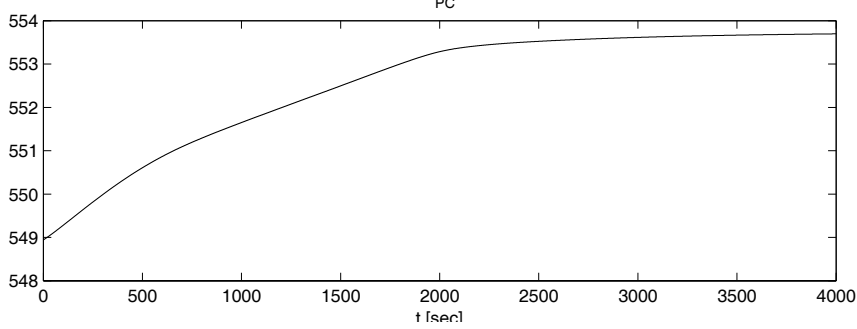

Fig. 2. Trajectories of $N, T_{P C}, T_{S G}, T_{P R}$
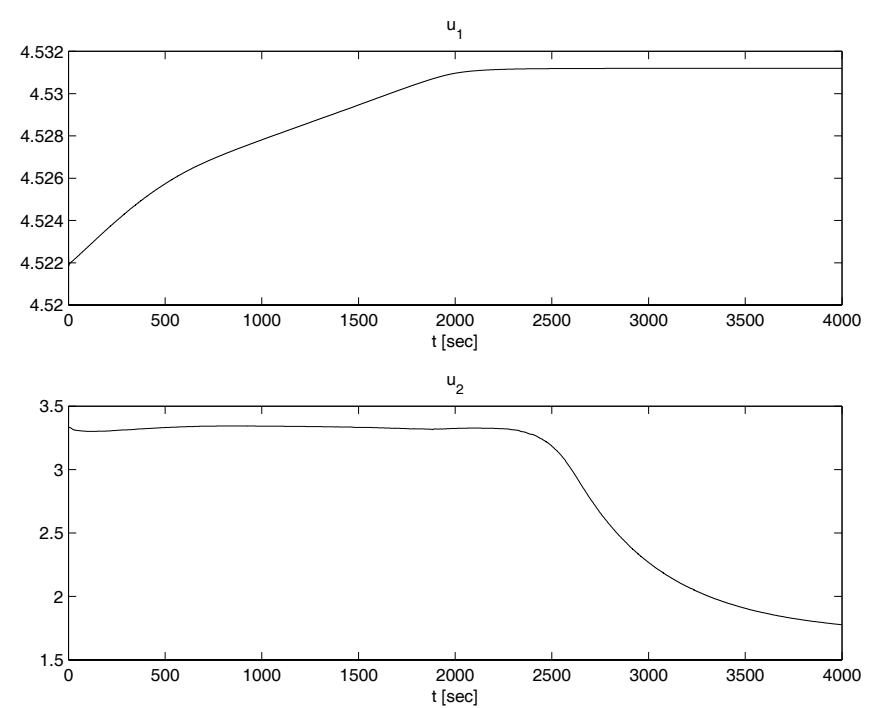

Fig. 3. Control inputs $u_{1}, u_{2}$ and mass flow $m_{P R}$

The weighting matrices in the cost function were chosen to be $Q_{1}=\operatorname{diag}(0.1,0.1,0.1,0.01), R=\operatorname{diag}(0.01,0.01)$. The feedback gain was updated at every $12 \mathrm{~s}(M=12)$. For the computation $2 \mathrm{~s}$ was allocated $(l=2)$.

The dynamic behavior of the system controlled by the modified MPC procedure can be seen in Figs. 2 and 3. These figures show that the algorithm is able to solve the control problem: the settling time of the neutron flux is acceptably small, the states and the control inputs satisfy the prescribed constraints.

The simulation was performed in MATLAB/SIMULINK by using LMI Control Toolbox. The computation time of the control gain at each time step was between $0.91 \mathrm{sec}$ and $1.42 \mathrm{sec}$ on a P4 $2.4 \mathrm{GHz}$ processor. Since these values are smaller than the allocated time $l \cdot T_{s}=2 \mathrm{sec}$ we can conclude that the modified control procedure is suitable for real-time application.

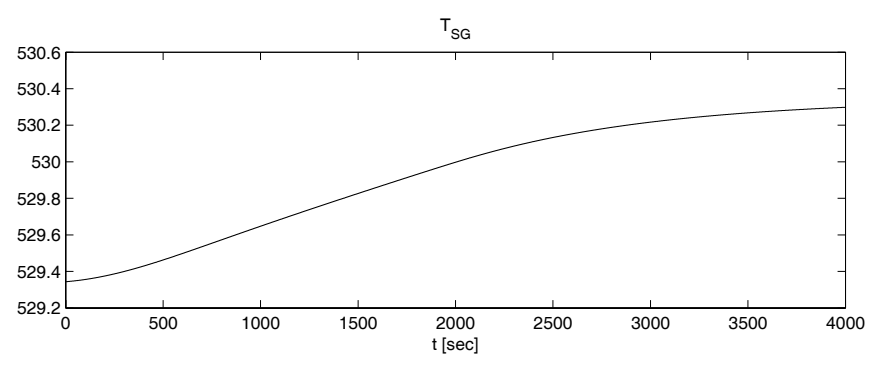

$\mathrm{T}_{\mathrm{PR}}$
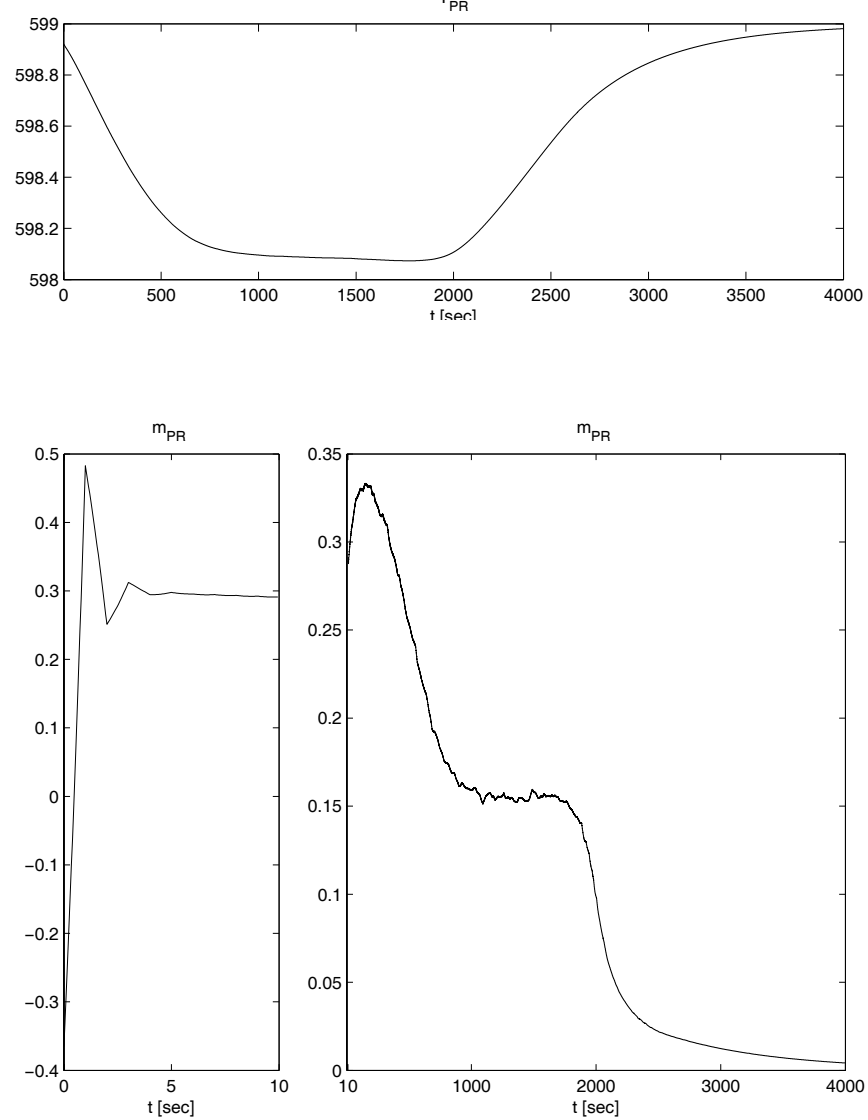

\section{Conclusions}

In this paper an LMI based model predictive regulator has been constructed for the primary circuit of a pressurized water nuclear power plant. During the control design it was shown that the dynamic behavior of the plant can be described well by a continuous hybrid LPV dynamics. Moreover, this model could be discretized so that the discrete time model obtained is also of LPV form with the same parameters as its continuous counterpart. Then, the discrete time hybrid model was embedded into a non-hybrid LPV structure, for which, effective control design methods exist. For the discrete LPV model we have successfully applied the LMI-based MPC algorithm proposed by [5]. Finally, a useful modification of the original control algorithm has been proposed to better suit it to our special needs. The dynamic behavior of the controlled system was investigated through numerical simulations and it has been found to satisfy the input and state constraints.

Further work will be directed towards two possible improvements of the proposed method. Firstly, the modeling of the real 


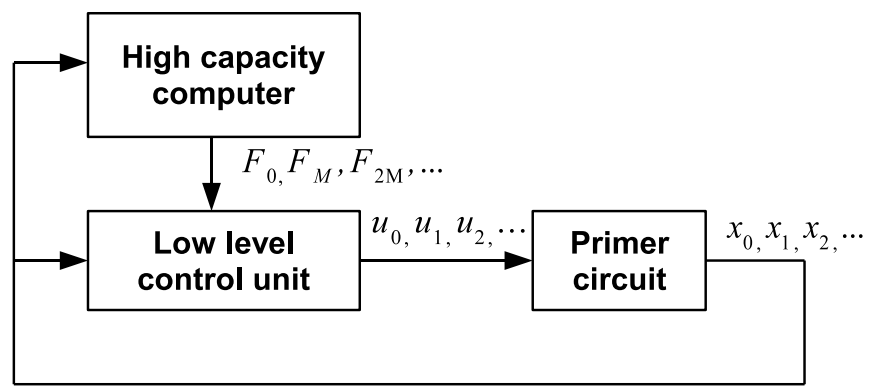

Fig. 4. Hardware architecture realizing the modified control policy

actuator dynamics of the neutronflux controller and secondly, the treatment of some parameters (especially $m_{S G}$ and $T_{S G, S W}$ ) as time-varying parameters in the LPV model.

\section{References}

1 Boyd S, Ghaoui L E, Feron E, Balakrishnan V, Linear matrix inequalities in system and control theory, SIAM, 1994.

2 Cuzzola F A, Geromel J C, Morari M, An improved approach for constrained robust model predictive control, Automatica 38 (2002), 1183-1189, DOI 10.1016/S0005-1098(02)00012-2.

3 Fazekas Cs, Szederkényi G, Hangos K M, A simple dynamic model of the primary circuit in VVER plants for controller design purposes, Nuclear Engineering and Design 237 (2006), 1071-1087, DOI 10.1016/j.nucengdes.2006.12.002.

4 Hangos K M, Cameron I T, Process modelling and model analysis, Academic Press, London, 2001.

5 Kothare M V, Balakrishnan V, Morari M, Robust constrained model predictive control using linear matrix inequalities, Automatica 32 (1996), no. 10, 1361-1379, DOI 10.1016/0005-1098(96)00063-5.

6 Maciejowski J M, Predictive control with constraints, Prentice Hall, 2002.

7 Mayne D Q, Rawlings J B, Rao C V, Scoakert P O M, Constrained model predictive control: Stability and optimality, Automatica 36 (2000), no. 3, 789-814, DOI 10.1016/S0005-1098(99)00214-9.

8 Péni T, Szederkényi G, Model predictive control of the hybrid primary circuit dynamics in a pressurized water nuclear power plant, Systems and Control Laboratory, SZTAKI, http://daedalus.scl.sztaki.hu/, June SCL.

9 Scherer C, Weiland S, Linear matrix inequalities in control, http://www.cs.ele.tue.nl/ SWeiland/ lmi.pdf, 2000. vers. 3.0.

10 Szabó Z, Gáspár P, Bokor J, Reference tracking of Wiener systems using dynamic inversion, Prep. 2005 international symposium on intelligent control, 2005, DOI 10.1109/.2005.1467184, (to appear in print). on CD, paper ID: WeA06.5.

11 Varga I, Szederkényi G, Hangos K M, Bokor J, Modeling and model identification of a pressurizer at the Paks nuclear power plant, 14th IFAC Symposium on System Identification - SYSID 2006, 2006, pp. 678-683. 